\title{
Correspondence
}

\section{Bacteria, arthritis, and skin lesions due to Kingella kingae}

Sir,

Kingella (Moraxella) kingae, a Gram-negative, nonencapsulated, nonmotile aerobic coccobacillus, is an occasional inhabitant of the upper respiratory tract. ${ }^{1}$ Rarely has it been associated with disease. Recently we cared for a young leukaemic patient in whom an episode of Kingella kingae septicaemia occurred. Illness in this child was associated with cutaneous and articular manifestations similar to those described in cases of sepsis caused by other members of the family of Neisserieae.

A 4-year-old boy with acute lymphocytic leukaemia in relapse was admitted with vesicular stomatitis, rash, and fever which had been treated with nystatin oral suspension for 2 days. He was listless but arousable and his rectal temperature was $38 \cdot 6^{\circ} \mathrm{C}$. There was a vesicular, pustular eruption affecting both lips, and the gums and tongue were coated with a yellowish-white friable plaque. Skin examination showed a vesicle (5 $\mathrm{mm}$ in diameter) on an erythematous base on the dorsum of the right hand, two erythematous papules $(1 \mathrm{~cm}$ in diameter) on the extensor surface of the left forearm, and an erythematous papule $(0.5 \mathrm{~cm}$ in diameter) on the plantar surface of the left foot. Gram stain of the oral plaque showed mixed bacterial flora, particularly Gram-negative bacilli; no budding cells or pseudohyphae were observed. The WBC was $0.77 \times 10^{9} / 1$ with $8 \%$ polymorphonuclears, $12 \%$ bandforms, $42 \%$ lymphocytes, $10 \%$ monocytes, $2 \%$ metamyelocytes, and $26 \%$ lymphoblasts; the platelet count was $<5.0 \times 10^{9} / 1$. Treatment was started with intravenously-administered gentamicin and carbenicillin.

On day 2 in hospital the left knee was noted to be erythematous, warm, swollen, and tender, but without evidence of a joint effusion. On day 3 two blood cultures taken on the day of admission were each reported to be growing a Gram-negative coccobacillus, subsequently confirmed to be $M$. (kingella) kingae, sensitive by disc diffusion susceptibility testing to chloramphenicol, tetracycline, gentamicin, carbenicillin, ampicillin, tobramycin, and polymixin B. The patient became afebrile on day 4. The skin lesions and left knee joint resolved, and repeat blood cultures were sterile during a 14-day course of the combined antibiotics.

Human strains of $K$. kingae have been isolated from nose, throat, blood, joint fluid, and bone. ${ }^{1}$ One isolate was obtained from the blood of a child with congenital heart disease and endocarditis. ${ }^{2}$ The organism has also been recovered from patients with pharyngitis, laryngitis, and an eyelid abscess, as well as from routine nose and throat swabs. ${ }^{3}$ In this case, the severe stomatitis, whether or not caused by $K$. kingae, may have provided the portal of entry for the organism. The cutaneous and articular manifestations might have been the result of haematogenously disseminated infection to skin and periarticular tissues. Such a syndrome is a recognised feature of disseminated infection due to Neisseria gonorrhoea and has been reported in an adult with sepsis caused by Moraxella osloensis. ${ }^{4}$ Moreover, Moraxella sp. have been reported to cause sepsis, ${ }^{5}$ and septic arthritis in children. ${ }^{6-7}$ Neisseria, Moraxella, and Kingella genuses are all members of the family of Neisserieae. The genus Kingella has recently been separated from the genus Moraxella by its haemolytic activity and characteristic biochemical reactions. However, definition of the exact role of $K$. kingae as a human pathogen will depend on further clinical experience, better laboratory recognition, and reporting.

\section{References}

1 Henriksen S D, Bovre K. Transfer of Moraxella kingae Henriksen and Bovre to the genus Kingella gen. nov. in the family Neisseriaeceae. Int $J$ Systematic Bacteriol 1976; 26: 447-50.

2 Christensen C E, Emmanouilides G C. Bacterial endocarditis due to 'Moraxella new species I'. $N$ Engl J Med $1967 ; 277$ : 803-4.

3 Henriksen S D. Corroding bacteria from the respiratory tract. I. Moraxella kingii. Acta Pathol Microbiol Scand B 1969; 75: 85-90.

- Lasser A E, Goldman E J. Moraxella bacteremia. Report of a case resembling gonococcemia with cutaneous manifestations. Cutis 1978; 21 : 657-9.

5 Butzler J P, Hansen W, Cadranel S, Henriksen S D. Stomatitis with septicemia due to Moraxella osloensis. JPediatr 1974; 84: 721-2

- Spahr R C. Letter: Septic arthritis due to Moraxella species. J Pediatr 1975; 86: 310.

7 Feigin R D, San Joaquin V, Middelkamp J N. Septic arthritis due to Moraxella osloensis. J Pediatr 1969; 75: 116-7.

David C Redfield, Gary D Overturf, Nadia EwIng, and Darleen Powars Department of Pediatrics, LAC-USC Medical Center, $1200 \mathrm{~N}$ State Street, Los Angeles, California 90033, USA

\section{Conservative care of the newborn baby}

Sir,

Much criticism has been made of Dr Hughes-Davies's report $^{1}$ on the comparison of conservative and intensive care of the newborn baby. Much of this criticism centres on the unsatisfactory nature of his comparisons of mortality rates for very low birthweight babies at Salisbury with those for England and Wales, his Wessex neighbours, and University College Hospital, London. ${ }^{2-3}$ 